\title{
Empirical Analysis of Colors in Indian Web Applications
}

\author{
Prof.R.B.Kulkarni \\ Assistant Professor (PhD) \\ Department of CSE. \\ Walchand Institute of Technology \\ Solapur University, Solapur, India
}

\author{
Supriya S. Ambarkar \\ Assistant Professor, MTech \\ Department of CSE \\ Walchand Institute of Technology \\ Solapur University, Solapur, India
}

\author{
Prof. Dr. S. K. Dixit \\ HOD, EXTC Dept, Ph.D. \\ Department of EXTC. \\ Walchand Institute of Technology \\ Solapur University, Solapur, India
}

\begin{abstract}
Color has potential to elicit emotions, there is very little research on color used in web applications and there impact. This is a qualitative analysis of the colors used in the most visited Indian websites. Web sites of various categories were analyzed to find out whether they met the various international standards for the use of color in web design. The basis for the analysis was the Web Content Accessibility Criteria for the "Use of Color" standardized by the World Wide Web Consortium (W3C) and use of the web safe color palette. A summary of the standards used as guidelines for this analysis has been provided along with the detailed analysis report and a tabular summary of the report. The report reveals that majority of the sites fail to meet the standards. It is concluded that there is a need for web designers to study and analyze the use of color, the need to satisfy the color standards, its advantages and follow the color standards accordingly. This paper gives an analysis report of how colors are affected in Indian Websites and its impact.
\end{abstract}

\section{Keywords}

Web content accessibility criteria, web safe, colors, analysis

\section{INTRODUCTION}

Color is an important asset in design of Web content, enhancing its aesthetic appeal, its usability, and its accessibility. Color is everywhere around us. Our visual and cognitive systems have adapted to perceive and process color information, which is contained in every visual stimulus we encounter [1][2]. Color information such as hue, brightness, and saturation has the potential to affect our perceptions, physiological reactions, emotional reactions or behavioral intentions [3]. However, some users have difficulty perceiving color. People with partial sight often experience limited color vision, and many older users do not see color well. In addition, people using text-only, limited-color or monochrome displays and browsers will be unable to access information that is presented only in color .For this purpose, it is important for web designers to follow certain rules regarding the choice of color schemes on websites. Also, all the information that is to be conveyed through difference of colors, must also be conveyed through other visual means[4].

\subsection{The Web Content Accessibility Guidelines:}

Web Content Accessibility Guidelines (WCAG) 2.0 covers a wide range of recommendations for making Web content more accessible. Following these guidelines will make content accessible to a wider range of people with disabilities, including blindness and low vision, deafness and hearing loss, learning disabilities, cognitive limitations, limited movement, speech disabilities, photosensitivity and combinations of these. Following these guidelines will also often make Web content more usable to users in general. For our analysis, we have used the following guidelines form WCAG 2.0 concerning the use of color in web design.

As per the following Guideline 1.4 Distinguishable: Make it easier for users to see and hear content including separating foreground from background. WCAG 2.0 [5][6] .

\subsubsection{Use of Color}

Color is not used as the only visual means of conveying information, indicating an action, prompting a response, or distinguishing a visual element. (Level A).Ensure that all users can access information that is conveyed by color differences, that is, by the use of color where each color has a meaning assigned to it. If the information is conveyed through color differences in an image (or other non-text format), the color may not be seen by users with color deficiencies. In this case, providing the information conveyed with color through another visual means ensures users who cannot see color can still perceive the information. Colors of distinguished text should provide 3:1 contrast with surrounding text colors and 4.5:1 contrast with the background color.

\subsubsection{Contrast (Minimum)}

The visual presentation of text and images of text has a contrast ratio of at least 4.5:1, except for the following: (Level AA).

Large Text: Large-scale text and images of large-scale text have a contrast ratio of at least $3: 1$;

Incidental: Text or images of text that are part of an inactive user interface component, that are pure decoration, that are not visible to anyone, or that are part of a picture that contains significant other visual content, have no contrast requirement.

Logotypes: Text that is part of a logo or brand name has no minimum contrast requirement. Provide enough contrast between text and its background so that it can be read by people with moderately low vision (who do not use contrastenhancing assistive technology).

\subsubsection{Contrast (Enhanced)}

The visual presentation of text and images of text has a contrast ratio of at least 7:1, except for the following: (Level AAA).

Large Text: Large-scale text and images of large-scale text have a contrast ratio of at least 4.5:1;

Incidental: Text or images of text that are part of an inactive user interface component, that are pure decoration, that are not 
visible to anyone, or that are part of a picture that contains significant other visual content, have no contrast requirement. Logotypes: Text that is part of a logo or brand name has no minimum contrast requirement.

Checkpoint 2.2 of the WCAG 1.0 requires that "foreground and background color combinations provide sufficient contrast when viewed by someone having color deficits, or when viewed on a black and white screen[5]".

\section{CALCUlating THE CONTRAST RATIO \\ 2.1 Contrast Ratio}

Measure the relative luminance of each letter (unless they are all uniform) using the formula: $\mathrm{L}=0.2126 * \mathrm{R}+0.7152 * \mathrm{G}$ $+0.0722 * \mathrm{~B}$ where $\mathrm{R}, \mathrm{G}$ and $\mathrm{B}$ are defined as:

- if RsRGB $<=0.03928$ then $\mathrm{R}=\mathrm{RsRGB} / 12.92$ else $\mathrm{R}=((\mathrm{RsRGB}+0.055) / 1.055) \wedge 2.4$

- if GsRGB $<=0.03928$ then $\mathrm{G}=\mathrm{GsRGB} / 12.92$ else $\mathrm{G}=((\mathrm{GsRGB}+0.055) / 1.055)^{\wedge} 2.4$

- if BsRGB $<=0.03928$ then $\mathrm{B}=\mathrm{BsRGB} / 12.92$ else $\mathrm{B}=((\mathrm{BsRGB}+0.055) / 1.055)^{\wedge} 2.4$

- and RsRGB, GsRGB, and BsRGB are defined as:

- $\quad$ RsRGB $=$ R8bit $/ 255$

- $\mathrm{GsRGB}=\mathrm{G} 8 \mathrm{bit} / 255$

- $\mathrm{BsRGB}=\mathrm{B} 8 \mathrm{bit} / 255$

\subsection{Calculating the color difference}

Color Difference Formula: Color difference is determined by the following formula:

(Maximum (Red value 1, Red value 2) - minimum (Red value 1 , Red value 2$)$ ) + (maximum (Green value 1 , Green value 2 ) - minimum (Green value 1 , Green value 2$))+($ maximum (Blue value 1 , Blue value 2) - minimum (Blue value 1 , Blue value 2 ))

The difference between the background color and the foreground color should be greater than 500 .

\subsection{Calculating the color brightness difference}

Color Brightness Formula: Color brightness is determined by the following formula:

$(($ Red value X 299) $+($ Green value X 587) $+($ Blue value $X$ 114)) / 1000

The difference between the background brightness, and the foreground brightness should be greater than 125 .

\subsection{Web Safe or Browser Safe Colors}

Web Safe, or Browser Safe palettes as they are also referred to, consist of 216 colors that display solid, non-dithered, and consistent on any computer monitor, or web browser, capable of displaying at least 8 -bit color (256 colors). The reason why this palette contains only 216 colors, instead of the maximum 256 colors, is that only 216 out of the basic 256 colors will display exactly the same on all computers. This discrepancy is similar to what happens when a Windows user opens a word processor document created on a Macintosh (or vice versa) and sees a lot of odd characters in place of the expected punctuation marks and other extended characters. While the basic character set of lowercase and upper case characters and numerals map identically on all platforms, each computer platform treats some extended characters, like ampersands, foreign currency symbols, accented characters, and so forth, differently. And so a proper curly quote mark on one computer platform might be a pound sign on another. This happens with colors as well, so what is gray on a Windows monitor, might display pale yellow on a Macintosh browser. In the same way that there are common characters and numbers that are consistent among computer platforms, so there are colors that display the same. These are what we call Web Safe colors. If you use browser safe colors, you can be sure that they will not dither on these machines and your pages will look the way that you intended them to look. It is true that most computers sold these days are sold with monitors and video cards that can support millions of colors. However, on a lot of these systems the default setting is at $640 \times 480$ pixels on the screen and 256 colors. Many people don't know that they can change that setting, let alone know how to do it[6][7].

\section{TOOLS FOR ANALYSIS}

\subsection{Color Contrast Analyzer, version 2.2}

It is primarily a tool for checking foreground \& background color combinations to determine if they provide good color visibility. It also contains functionality to create simulations of certain visual conditions such as color blindness. Determining "color visibility" is based on the Contrast Ratio algorithm, suggested by the $\mathrm{W} 3 \mathrm{C}$. The contrast ratio is now a W3C recommendation (December 2008), to help determine whether or not the contrast between two colors can be read by people with color blindness or other visual impairments. The Contrast Analyzer (CA) is useful to help determine, in particular, the legibility of text on a web page and the legibility of image based representations of text.

\subsection{Chart of the $\mathbf{2 1 6}$ web safe colors}

A chart of the 216 web safe colors was used to find out whether the color used on the web site is web safe.

\section{COLOR ANALYSIS REPORT OF WEB SITES}

Color is a powerful method from which you can attract the people very efficiently. When people visiting an art exhibition, why do they spent for 30 minutes in front of one painting and only 10 seconds in front of another? What they feel in front of one painting? Even when two paintings are of the same nature, painted in the same period, in the same style, and will suppose by the same artist. So we can attract through a powerful combination of colors and we make our visitors to visit our website repeatedly[8].

Web site design's aesthetics when cool color combinations (blue-light blue), as opposed to warm color combinations (red-orange), are used; direct effects of classical aesthetic dimensions (e.g., cleanliness) on expressive aesthetics items (e.g., creativity); and no effects of gender on either set of aesthetics[9].

'Use colors with high contrast between the text and the background. Optimal legibility requires black text on white 
background (so-called positive text). White texton a black background (negative text) is almost as good. Although the contrast ratio is the same as for positive text, the inverted color scheme throws people off a little and slows their reading slightly. Legibility suffers much more for color schemes that make the text any lighter than pure black, especially if the background is made any darker than pure white'[10].

Based on the Guideline 1.4 Distinguishable of WCAG 2.0

(1.4.1 [Use of Color], 1.4.3 [Contrast Minimum], and 1.4.6[Contrast Enhanced]) [11][12].

\subsection{Rediff (portal)}

\section{SC 1.4.1:}

- Difference of color is provided to distinguish links from ordinary text.

- All links are visually enhanced on hover or focus by text becoming bold and change of mouse pointer type.

- Text alternatives are provided for image links. Hence, they do not solely depend on color and change of mouse pointer type for distinguishability or for indicating the presence of a link.

- The contrast between distinguished texts is more than 3:1 and that between foreground and

SC 1.4.3: and SC 1.4.6 background colors is more than 4.5:1.

The following Fig 1 shows us the screenshot of rediff.com web site which gives us the details regarding colors.

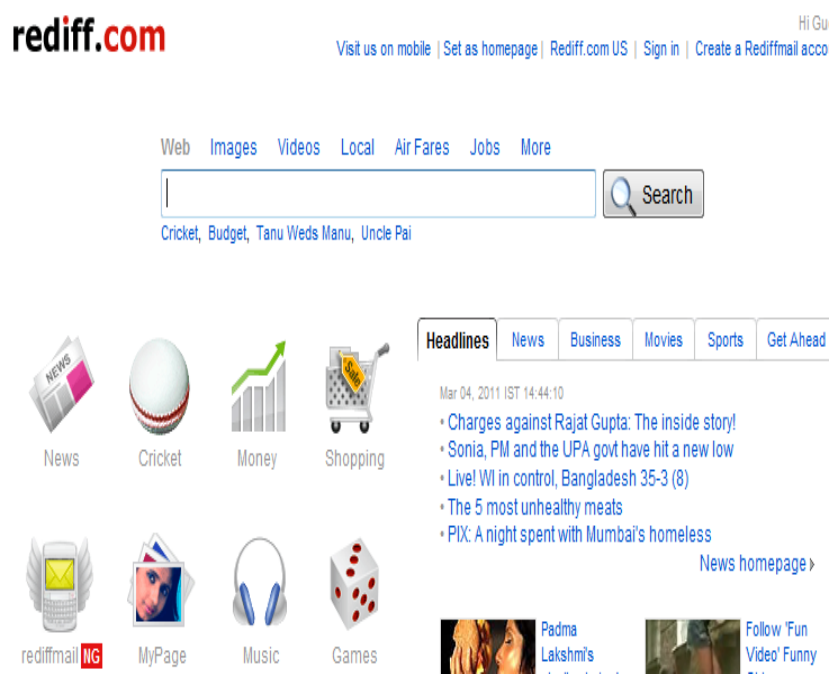

Fig 1: Screenshots of rediff.com website

Color difference and Brightness difference: Foreground:\#0055CC Background:\#FFFFFF

color difference:476/brightness difference: 182

- The difference in brightness between the two colors is sufficient. The threshold is 125 , and the result of the foreground and background colors is 182 .

- The difference in color between the two colors is not sufficient.

- The threshold is 500, and the result of the foreground and background colors is 476 .

Color Blindness: Normal: 476, 182, Protanopia: 467, 185,

Deuteranopia: 495, 200, Tritanopia: 455, 18
Luminosity:

Foreground:\#0055CC Background:\#FFFFFF

The contrast ratio is: 6.6:1

Text passed at Level AA. Large text passed at Level AA

Text failed at Level AAA. Large text passed at Level AAA

Color Blindness: Normal: 6.6:1, Protanopia: 8.0:1, Deuteranopia: 9.1:1, Tritanopia: 6.4:1

Web safe colors:

- $\quad \# 0055 C$ C: not web safe.

- Closest web safe color is \#0033CC.

\subsection{IndiaTimes (portal): \\ SC 1.4.1:}

- Difference of color is provided to distinguish links from ordinary text.

- No visual cues apart from change of mouse pointer type are provided for links when focused or hovered upon.

- Text alternatives are provided for image links. Hence, they do not solely depend on color and change of mouse pointer type for distinguishability or for indicating the presence of a link.

- The contrast between distinguished texts is more than $3: 1$ and that between foreground and background colors is more than $4.5: 1$.

\section{SC 1.4.3: and SC 1.4.6}

The following Fig 2 shows us the screenshot of indiatimes.com web site which gives us the details regarding colors.

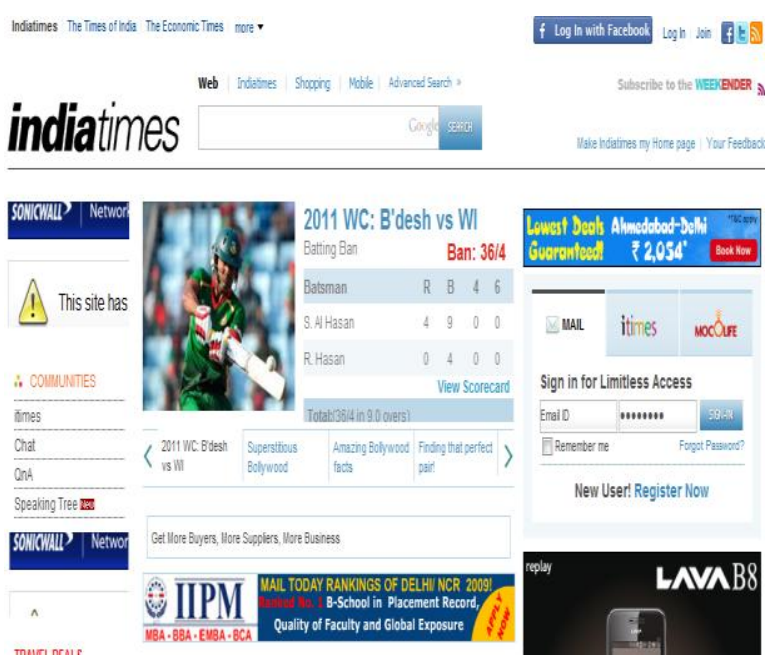

Fig 2 : Screenshots of indiatimes.com website

Color difference and Brightness difference:

Foreground:\#458EBD Background:\#FFFFFFB color difference:365/brightness difference: 130 .

The difference in brightness between the two colors is sufficient. The threshold is 125 , and the result of the foreground and background colors is 130 .

The difference in color between the two colors is not sufficient. The threshold is 500, and the result of the foreground and background colors is 365 .

Color Blindness: Normal: 365, 130, Protanopia: 332, 123, Deuteranopia: 356, 133, Tritanopia: 358, 129. 
Foreground:\#4D4D4D Background:\#FFFFFF color difference:534/brightness difference: 178

The difference in brightness between the two colors is sufficient. The threshold is 125, and the result of the foreground and background colors is 178 .

The difference in color between the two colors is sufficient. The threshold is 500, and the result of the foreground and background colors is 534 .

Color Blindness: Normal: 534, 178,Protanopia: 536, 179,Deuteranopia: 536, 178,Tritanopia: 533, 178.

Luminosity:

Foreground:\#458EBD

Background:\#FFFFFF The contrast ratio is: 3.6:1

Text failed at Level AA. Large text passed at Level AA

Text failed at Level AAA. Large text failed at Level AAA

Color Blindness: Normal: 3.6:1,Protanopia:

3.7:1,Deuteranopia: 4.2.1, Tritanopia: 3.6:1

Foreground:\#4D4D4D Background:\#FFFFFF

The contrast ratio is: $8.5: 1$

Text passed at Level AA. Large text passed at Level AA

Text passed at Level AAA. Large text passed at Level AAA

Color Blindness: Normal: 8.5:1, Protanopia: 8.5:1, Deuteranopia: 8.4:1,Tritanopia: 8.5:1

\section{Web safe colors:}

- \#458EBD: not web safe

- \#4D4D4D: not web safe. Closest web safe color is \#4B4B4B.

\subsection{IRCTC (Railway Reservations) \\ SC 1.4.1:}

- Difference of color is provided to distinguish links from ordinary text.

- All links are visually enhanced on hover or focus by underlining and text becoming bold and change of mouse pointer type.

- Text alternatives are not provided for image links. Hence, they solely depend on color and change of mouse pointer type for distinguishability or for indicating the presence of a link.

- The contrast between distinguished texts is more than $3: 1$ and that between foreground and background colors is more than 4.5:1.

\section{SC 1.4.3: and SC 1.4.6}

The following Fig 1 shows us the screenshot of irctc.com web site which gives us the details regarding colors.

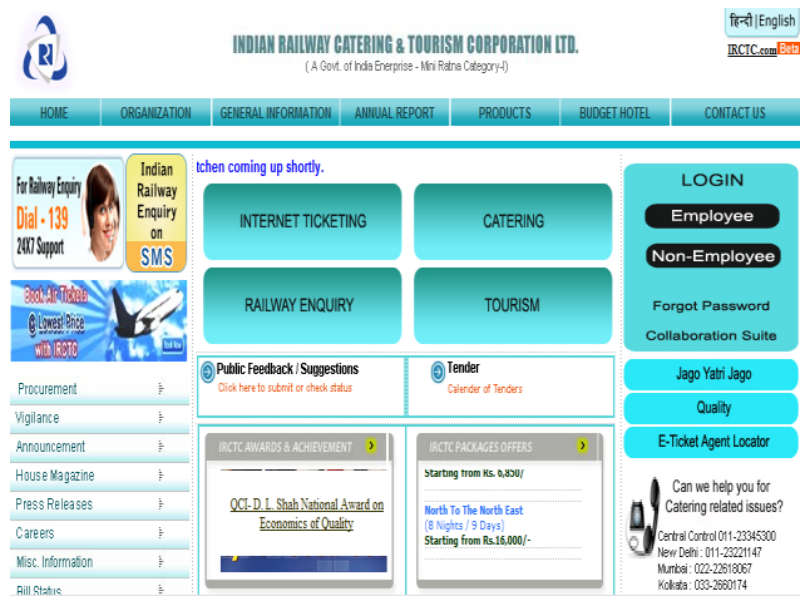

Fig 3 : Screenshots of irctc.com website

\section{Color difference and Brightness difference:}

Foreground:\#000000 Background:\#F1F1F1 color difference:723/brightness difference: 241

The difference in brightness between the two colors is sufficient. The threshold is 125 , and the result of the foreground and background colors is 241. The difference in color between the two colors is sufficient. The threshold is 500 , and the result of the foreground and background colors is 723.

Color Blindness: Normal: 723, 241,Protanopia: 722, 240, Deuteranopia: 723, 240, Tritanopia: 722, 240.

Foreground:\#565656 Background:\#2680BA color difference: $190 /$ brightness difference: 21

The difference in brightness between the two colors is not sufficient. The threshold is 125 , and the result of the foreground and background colors is 21 . The difference in color between the two colors is not sufficient. The threshold is 500 , and the result of the foreground and background colors is 190.

Color Blindness: Normal: 190, 21,Protanopia: 132, 29, Deuteranopia: 124, 15, Tritanopia: 204, 23

Luminosity:

Foreground:\#000000 Background:\#F1F1F1

The contrast ratio is: $18.6: 1$

Text passed at Level AA. Text passed at Level AAA

Large text passed at Level AA. Large text passed at Level AAA

Color Blindness: Normal: 18.6:1,Protanopia: 18.5:1, Deuteranopia: 18.5:1,Tritanopia: 18.5:1.

Foreground:\#565656 Background:\#2680BA

The contrast ratio is: $1.7: 1$

Text failed at Level AA. Text failed at Level AAA

Large text failed at Level AA. Large text failed at Level AAA Color Blindness: Normal: 1.7:1, Protanopia: 1.6:1, Deuteranopia: 1.3:1,Tritanopia: 1.7:1.

Web safe colors:

- \#F1F1F1: not web safe. Closest web safe color is \#DDDDDD

- \#565656: not web safe. Closest web safe color is \#4B4B4B.

- \#2680BA: not web safe. Closest web safe color is \#3366CC.

\subsection{BharatStudent (Social Networking)}

SC 1.4.1:

- Difference of color is provided to distinguish links from ordinary text.

- No visual cues apart from change of mouse pointer type are provided for links when focused or hovered upon.

- Text alternatives are provided for image links. Hence, they do not solely depend on color and change of mouse pointer type for distinguishability or for indicating the presence of a link.

- The contrast between distinguished texts is more than $3: 1$ and that between foreground and background colors is more than 4.5:1.

\section{SC 1.4.3: and SC 1.4.6}

The following Fig 4 shows us the screenshot of bharatstudent.com web site which gives us the details regarding colors. 


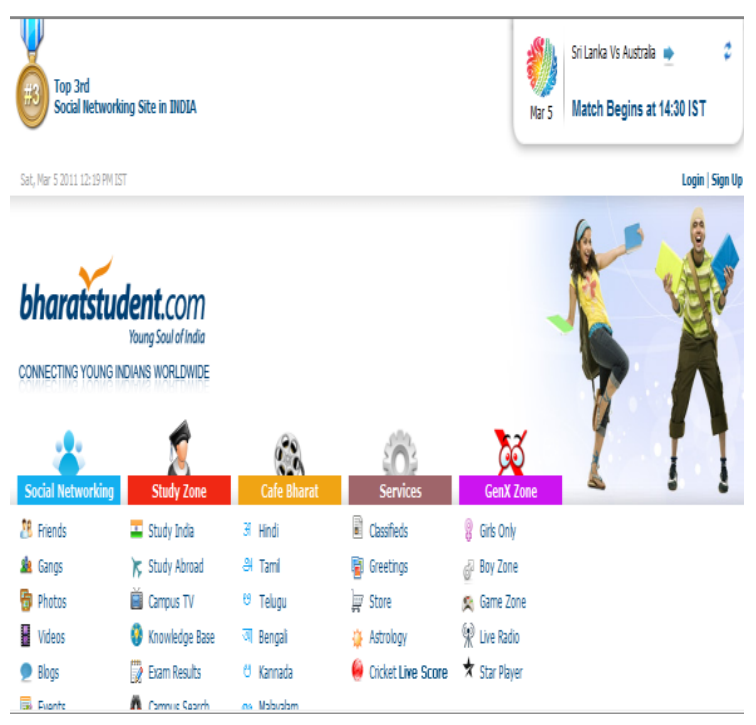

Fig 4 : Screenshots of bharatstudent.com website

Color difference and Brightness difference:

Foreground:\#383838 Background:\#FFFFFF

color difference:597/brightness difference: 199

The difference in brightness between the two colors is sufficient. The threshold is 125, and the result of the foreground and background colors is 199 .

The difference in color between the two colors is sufficient. The threshold is 500 , and the result of the foreground and background colors is 597 .

Color Blindness: Normal: 597, 199,Protanopia: 599, 200, euteranopia: 599, 199,Tritanopia: 595, 199

Foreground:\#004A7F Background:\#FFFFFF color difference:564/brightness difference: 198

The difference in brightness between the two colors is sufficient. The threshold is 125, and the result of the foreground and background colors is 198 .

The difference in color between the two colors is sufficient. The threshold is 500, and the result of the foreground and background colors is 564

Color Blindness: Normal: 564, 198, Protanopia: 551, 199, Deuteranopia: 581, 214,Tritanopia: 515, 195

\section{Luminosity:}

Foreground:\#383838 Background:\#FFFFFF

The contrast ratio is: $11.7: 1$

Text passed at Level AA. Text passed at Level AAA

Large text passed at Level AA. Large text passed at Level AAA

Color Blindness: Normal: 11.7:1,Protanopia: 11.8:1, Deuteranopia: 11.6:1,Tritanopia: 11.7:1

Foreground:\#004A7F Background:\#FFFFFF

The contrast ratio is: $9.2: 1$

Text passed at Level AA. Text passed at Level AAA

Large text passed at Level AA. Large text passed at Level AAA

Color Blindness: Normal: 9.2:1, Protanopia: 11.1:1, Deuteranopia: 13.1:1,Tritanopia: 8.5:1.

\section{Web safe colors:}

- \#383838: not web safe. Closest web safe color is \#4B4B4B.
- \#004A7F: not web safe. Closest web safe color is \#003366.

\subsection{Sify (portal) \\ SC 1.4.1:}

- All links are visually enhanced on hover or focus by underlining and change of color and change of mouse pointer type.

- The contrast between distinguished texts is more than 3:1 and that between foreground and background colors is more than 4.5:1.

- The links provided by images do not have any text alternative. Hence, they solely depend on color and change of mouse pointer type for distinguishability or for indicating the presence of a link.

\section{SC 1.4.3: and SC 1.4.6}

The following Fig 5 shows us the screenshot of sify.com web site which gives us the details regarding colors.

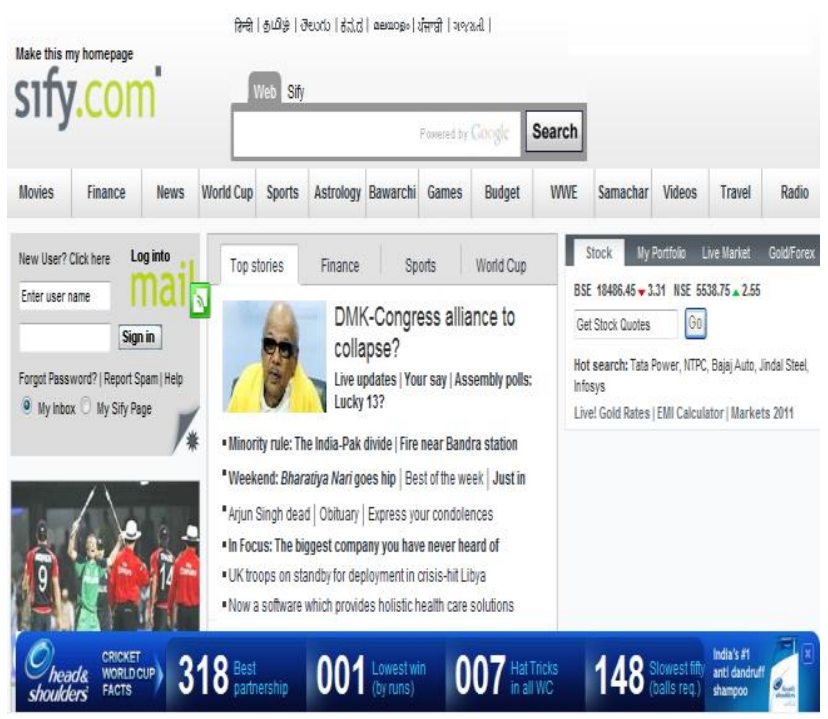

Fig 5 : Screenshots of sify.com website

Color difference and Brightness difference:

Foreground:\#666666 Background:\#FFFFFF

color difference:459/brightness difference: 153

The difference in brightness between the two colors is sufficient. The threshold is 125 , and the result of the foreground and background colors is 153 .

The difference in color between the two colors is not sufficient. The threshold is 500, and the result of the foreground and background colors is 459 .

Color Blindness: Normal: 459, 153,Protanopia: 461, 154, Deuteranopia: 461, 153,Tritanopia: 458, 153

Foreground:\#000000 Background:\#DCDCDC

color difference:660/brightness difference:220

The difference in brightness between the two colors is sufficient. The threshold is 125 , and the result of the foreground and background colors is 220 .

The difference in color between the two colors is sufficient. The threshold is 500, and the result of the foreground and background colors is 660

Color Blindness: Normal: 660, 220,Protanopia: 658, 219, Deuteranopia: 659, 219,Tritanopia: 658, 219 
Foreground:\#666666 Background:\#F8FAFB

color difference:443/brightness difference: 147

The difference in brightness between the two colors is sufficient. The threshold is 125 , and the result of the foreground and background colors is 147 .

The difference in color between the two colors is not sufficient. The threshold is 500, and the result of the foreground and background colors is 443 .

Color Blindness: Normal: 443, 147,Protanopia: 448, 149, Deuteranopia: 446, 147, Tritanopia: 442, 147.

\section{Luminosity}

Foreground:\#666666 Background:\#FFFFFF

The contrast ratio is: $5.7: 1$

Text passed at Level AA. Text failed at Level AAA

Large text passed at Level AA. Large text passed at Level

AAA

Color Blindness: Normal: 5.7:1, Protanopia: 5.8:1,

Deuteranopia: 5.7:1, Tritanopia: 5.8:1

Foreground:\#000000 Background:\#DCDCDC
The contrast ratio is: $15.3: 1$

Text passed at Level AA. Text passed at Level AAA

Large text passed at Level AA. Large text passed at Level AAA

Color Blindness: Normal: 15.3:1,Protanopia: 15.2:1, Deuteranopia: 15.2:1,Tritanopia: 15.3:1.

Foreground:\#666666 Background:\#F8FAFB

The contrast ratio is: 5.5:1

Text passed at Level AA. Text failed at Level AAA

Large text passed at Level AA. Large text passed at Level AAA

Color Blindness: Normal: 5.5:1, Protanopia: 5.5:1, Deuteranopia: 5.4:1, Tritanopia: 5.5:1.

\section{Web safe colors:}

- 666666: web safe.

- \#F8FAFB: not web safe. Closest web safe color is \#FFFFFF.

- \#DCDCDC: not web safe. Closest web safe color is \#DDDDDD.

\section{REPORT SUMMARY}

Following is the summary report of Indian web sites colors:

\begin{tabular}{|c|c|c|c|c|c|}
\hline $\begin{array}{l}\text { Sr. } \\
\text { no }\end{array}$ & $\begin{array}{l}\text { Domain Name } \\
\text { And type }\end{array}$ & Criteria 1.4.1 & $\begin{array}{c}\text { Criteria 1.4.3 and Criteria } \\
\text { 1.4.6 }\end{array}$ & $\begin{array}{l}\text { Web safe/ } \\
\text { Browser } \\
\text { safe colors }\end{array}$ & $\begin{array}{c}\text { Results for } \\
\text { Color Blindness }\end{array}$ \\
\hline 1. & $\begin{array}{l}\text { Rediff. Com } \\
\quad \text { (portal) }\end{array}$ & Passed & $\begin{array}{c}\text { Failed (Color difference } \\
\text { insufficient. Small text fails } \\
\text { at Level AAA ) }\end{array}$ & No & Failed \\
\hline 2 & $\begin{array}{l}\text { Indiatimes.com } \\
\quad \text { (portal) }\end{array}$ & $\begin{array}{c}\text { Failed (no visual } \\
\text { enhancement for } \\
\text { distinguishing links) }\end{array}$ & $\begin{array}{l}\text { Failed (Color difference is } \\
\text { insufficient. } \\
\text { Text fails at level AA and } \\
\text { AAA.) }\end{array}$ & No & Failed \\
\hline 3 & $\begin{array}{c}\text { IRCTC.co.in } \\
\text { (railway reservations } \\
\text { portal) }\end{array}$ & $\begin{array}{l}\text { Failed (no text alternative } \\
\text { for image links) }\end{array}$ & $\begin{array}{l}\text { Failed (Color difference is } \\
\text { insufficient. Text fails at all } \\
\text { levels.) }\end{array}$ & No & Failed \\
\hline 4 & $\begin{array}{c}\text { Bharatstudent.com } \\
\text { (social networking site) }\end{array}$ & $\begin{array}{c}\text { Failed (no visual } \\
\text { enhancement to distinguish } \\
\text { links) }\end{array}$ & Passed & No & Passed \\
\hline 5 & $\begin{array}{l}\text { Sify.com } \\
\text { (portal) }\end{array}$ & $\begin{array}{l}\text { Failed (no text alternative } \\
\text { for image links) }\end{array}$ & $\begin{array}{c}\text { Failed (Color difference is } \\
\text { insufficient. Text fails at } \\
\text { level AAA.) }\end{array}$ & No & Failed \\
\hline
\end{tabular}

Table 1 : Analysis Report of Indian Web sites 


\section{CONCLUSION}

We have studied and analyzed the different categories of web sites (portals). Color is a very important feature of a Web site. Result reveals that web application color is significant determinant for web site trust and satisfaction. From the practical perspective studied from this paper it is very important to pay attention to color entities in the web applications. As color have a strong impact on the users to decide to stay or stick to the web applications. One of the first things people notice about your Web site is color combination and how much time he is going to spent.

When they visit your Web site color makes a one powerful tool to handle human beings brain. Here different web domains and how those web domains are follows through the criteria 1.4.1, 1.4.3, 1.4.6. Colors whether web safe or browser safe and finally result of color blindness has given. This paper gives us an important analysis report of Indian web site colors.

\section{REFERENCES}

[1] Elliot, A.J., Maier, M.A., 2007. Color and psychological functioning. Current Directions in Psychological Science $16(5), 250-254$.

[2] Kaya, N., Epps, H.H., 2004. Relationship between color and emotion: a study of college students. College Student Journal 38 (3), 396-405.

[3] Valdez, P., Mehrabian, A., 1994. Effects of color on emotions. Journal of Experimental Psychology 123 (4), 394-409.

[4] Dianne Cyra, MilenaHeadb, HectorLarios, Colour appealinwebsitedesignwithinandacrosscultures: A multi- methodevaluation , Int. J. Human-Computer Studies 68 (2010) 1-21.

[5] Web Content Accessibility Guidelines 2.0 http://www.w3.org/TR/2008/REC-WCAG20-20081211/

[6] Thomas Mandl,Maximilian Eibl , An Empirical assessment of Colours Use on the WWW.

[7] D. Karatzas, A. Antonacopoulos , Colour text segmentation in web images based on human perception Image and Vision Computing, 25 (2007) 564-577.

[8] Gitte Lindgaard, Aesthetics, Visual Appeal, Usability and User Satisfaction: What Do the User's Eyes Tell the User's Brain?, Australian Journal of Emerging Technologies and Society Vol. 5, No. 1, 2007, pp: 1-14.

[9] Constantinos K. Coursaris, Sarah J. Swierenga, Ethan Watrall, An Empirical Investigation of Color Temperature and Gender Effects on Web Aesthetics, JUS, Vol. 3, Issue 3, May 2008, pp. 103-117.

[10] Richard H. Hall, Patrick Hanna, The Impact of Web Page Text-Background Color Combinations on Readability, Retention, Aesthetics, and Behavioral Intention, 2004.

[11] Dianne Cyr,Milena Head, Hector Larios, Colour appeal in website design within and across cultures A multimethod evaluation, International Journal on Human Computer Studies 68 pp 1-21, 2010.

[12] Helan Petrie, Cristophar Power, Omar Khair ,David Swallow, D3.5 : Evaluation of Colour Contrast Module, Sixth Framework Programme Information Society Technology Priority, pp 1-34,2008. 